\title{
Rapid identification of causative insertions underlying Medicago truncatula Tnt1 mutants defective in symbiotic nitrogen fixation from a forward genetic screen by whole genome sequencing
}

Vijaykumar Veerappan ${ }^{1}$, Mehul Jani ${ }^{1}$, Khem Kadel $^{1}$, Taylor Troiani ${ }^{1}$, Ronny Gale ${ }^{1}$, Tyler Mayes ${ }^{1}$, Elena Shulaev ${ }^{1}$, Jiangqi Wen², Kirankumar S. Mysore ${ }^{2}$, Rajeev K. Azad ${ }^{1,3}$ and Rebecca Dickstein ${ }^{1 *}$

\begin{abstract}
Background: In the model legume Medicago truncatula, the near saturation genome-wide Tnt1 insertion mutant population in ecotype R108 is a valuable tool in functional genomics studies. Forward genetic screens have identified many Tnt1 mutants defective in nodule development and symbiotic nitrogen fixation (SNF). However, progress toward identifying the causative mutations of these symbiotic mutants has been slow because of the high copy number of Tnt 1 insertions in some mutant plants and inefficient recovery of flanking sequence tags (FSTs) by thermal asymmetric interlaced PCR (TAIL-PCR) and other techniques.

Results: Two Tht1 symbiotic mutants, NF11217 and NF10547, with defects in nodulation and SNF were isolated during a forward genetic screen. Both TAIL-PCR and whole genome sequencing (WGS) approaches were used in attempts to find the relevant mutant genes in NF11217 and NF10547. Illumina paired-end WGS generated 16 Gb of sequence data from a 500 bp insert library for each mutant, yielding $40 \mathrm{X}$ genome coverage. Bioinformatics analysis of the sequence data identified 97 and 65 high confidence independent Tnt1 insertion loci in NF11217 and NF10547, respectively. In comparison to TAIL-PCR, WGS recovered more Tnt1 insertions. From the WGS data, we found Tht1 insertions in the exons of the previously described PHOSPHOLIPASE C (PLC)-like and NODULE INCEPTION (NIN) genes in NF11217 and NF10547 mutants, respectively. Co-segregation analyses confirmed that the symbiotic phenotypes of NF11217 and NF10547 are tightly linked to the Tnt1 insertions in PLC-like and NIN genes,

respectively.
\end{abstract}

Conclusions: In this work, we demonstrate that WGS is an efficient approach for identification of causative genes underlying SNF defective phenotypes in M. truncatula Tnt1 insertion mutants obtained via forward genetic screens.

Keywords: Whole genome sequencing, Medicago truncatula, Symbiotic nitrogen fixation, Nodulation, Tnt1 mutants, Forward genetics, TAIL-PCR, NIN, PLC-like, DNF2

\footnotetext{
* Correspondence: beccad@unt.edu

'Department of Biological Sciences, University of North Texas, 1155 Union

Circle \#305220, Denton, TX 76203, USA

Full list of author information is available at the end of the article
} 


\section{Background}

Symbiotic nitrogen fixation (SNF) in legumes takes place in nodules, specialized organs that initiate by differentiation of root cells during invasion of the root by soil bacteria collectively known as rhizobia. Ultimately, the rhizobia are deposited within host plant cells, separated by a plant-derived membrane. Within mature nodules, rhizobia convert atmospheric nitrogen to bioavailable forms which it exchanges for photoassimilates from the plant host. This mutually beneficial symbiosis provides legumes and subsequent crops with a renewable nitrogen source. Huge changes in gene expression in both the plant and rhizobia are observed during the differentiation to functional nodules. Genetics has uncovered many rhizobial genes required for SNF, but many plant genes essential to SNF have yet to be discovered [1]. Significant progress has been made via forward genetic studies in identifying essential plant genes required for the early Nod-factor signaling pathway [2]. This pathway initiates nodule-specific plant gene expression in response to rhizobial lipochitooligosaccharide molecules called nodulation (Nod) factors. Although some genes have been discovered that are required for steps after Nod-factor signaling, many still await discovery and characterization.

Forward genetics can identify new genes essential for SNF, not biased by our presuppositions. Successful forward genetics projects require an efficient mutagenesis technique to induce sufficient number of random mutations to saturate the genome and also fast and robust methods to identify the causative mutations in genes underlying mutant phenotypes.

Medicago truncatula (barrel medic) is an excellent model to study legume-rhizobia interactions during SNF because of its ease of laboratory manipulation and the availability of extensive genetic and genomic resources $[3,4]$. In M. truncatula, a large collection of genomewide insertion mutants has been developed using the tobacco Tht1 (transposable element of Nicotiana tabacum) retrotransposon [5]. There are 21,000 Tnt1 insertion lines containing approximately 520,000 random insertions available as a community resource for functional genomics studies [6]. The Tht1 transposon is a $5.3 \mathrm{~Kb}$ long autonomous copia-like element first isolated from tobacco (N. tabacum) [7]. Tnt1 sequences encode a capsid-related protein (GAG), a protease (PR), an integrase (INT), a reverse transcriptase (RT) and ribonuclease $\mathrm{H}(\mathrm{RH})$, and contain a 610 bp longterminal repeat (LTR) flanking each end of Tnt1 [8]. Tnt1 transposes autonomously by a copy-and-paste mechanism through an RNA intermediate during somatic embryogenesis in tissue culture, thereby causing large numbers of random insertions across the genome $[5,8,9]$. Previous studies in M. truncatula, based on Southern blot analyses and flanking sequence tags (FSTs) isolated by TAIL-PCR, established an average of 25 insertions per Tnt1 line, with individual lines containing 6 to 59 independent insertions [5]. Tht1 has also been successfully used in large-scale genome-wide insertional mutagenesis of several other heterologous plant species including lettuce [10], soybean [11] and potato [12].

High-copy numbers of Tht1 insertions in the M. truncatula mutant lines are advantageous because fewer lines need to be generated to saturate the genome and fewer plants need to be screened in forward genetic screens to find mutants defective in pathways of interest. Near-saturation mutagenesis also increases the success rates of reverse genetic screening to find Tht 1 insertions in genes of interest [6]. However, high numbers of Tht1 insertions still pose significant challenges for forward genetic screens with recovery of FSTs a rate-limiting step. Traditional methods of FST identification, such as TAIL-PCR, adapter ligation PCR and plasmid rescue techniques, are not always efficient at identifying all the FSTs in individual Tht1 mutants.

In $M$. truncatula, numerous mutants that are defective in nodule development and symbiotic nitrogen fixation were identified by forward genetic screening of Tnt1 insertion populations [13]. Despite the near-saturation mutagenesis of Tnt 1 insertion lines and the collection of mutants available for forward genetic screens, causative mutations for only a limited number of lines have been identified in this population by forward genetics $[14,15]$. Whole genome sequencing (WGS) has revolutionized the identification of insertion mutations, caused by insertion of transposons or transfer-DNAs (T-DNAs), underlying the defective phenotypes of mutants from diverse organisms [16-19].

In this report, we demonstrate the successful use of WGS technology for the rapid identification of causative genes of $M$. trunctaula Tnt1 mutants defective in nodulation identified from a forward genetic screen. We compare WGS results for Tnt1 FSTs with those obtained by TAIL-PCR and find that the WGS approach is more efficient.

\section{Results and discussion}

Forward genetic screening for $M$. truncatula mutants with nodulation defects

To identify novel genes required for nodule development and SNF, we performed a forward genetic screen using the Tnt1 insertion population in the M. truncatula R108 ecotype background [5]. Primary screening for mutants was conducted at the $M$. truncatula community mutant screening workshops at the S. R. Noble Foundation. Plants were grown on a mixture of perlite and sand (3:1) and regularly irrigated with media containing low nitrate $(0.5 \mathrm{mM} \mathrm{KNO})_{3}$. Plants were inoculated with rhizobial 
strain Sinorhizobium meliloti Sm1021 [20] and screening was performed 4 weeks post inoculation (Fig. 1). When grown under low nitrate and symbiotic conditions, R108 wild-type (WT) plant shoots are green with roots having large ovoid pink nodules. The pink color of WT nodules is an indicator of efficient $\mathrm{N}_{2}$ fixation, caused by the abundant leghemoglobin protein [21]. In contrast, most SNF mutants show restricted shoot growth with anthocyanin accumulation in aerial parts and have small bumps (Nod+/-), spherical white nodules (Fix-) or pinkish white $($ Fix $+/-)$ nodules on their roots instead of distinct pink nodules. Approximately twelve $R_{1}$ plants per Tnt1 line were screened for defective symbiotic phenotypes and categorized by the severity of the defects observed [13] to identify putative mutants.

Secondary screening was performed to confirm phenotypes of putative mutants, using an aeroponic system as described previously $[22,23]$. The rhizobial strain $S$. meliloti Rm41 hemA:lac $Z$ was used in the secondary screening because $R m 41$ is more efficient in inducing nodulation and SNF in the R108 ecotype [24]. At 15 dpi, mutant plants were characterized for nitrogen deficiency phenotypes: leaf color and nodule shape, nodule color and rhizobial occupancy of sectioned nodules using X-Gal (5-Bromo-4-chloro-3-indolyl-P-galactopyranoside) staining for the lac $Z$ gene contained in the rhizobial strain. Mutants with clear nodule defects and SNF phenotypes were selected for further characterization. Among the mutants chosen for further characterization were those from lines NF11217 and NF10547, both with Nod + Fix- phenotypes.

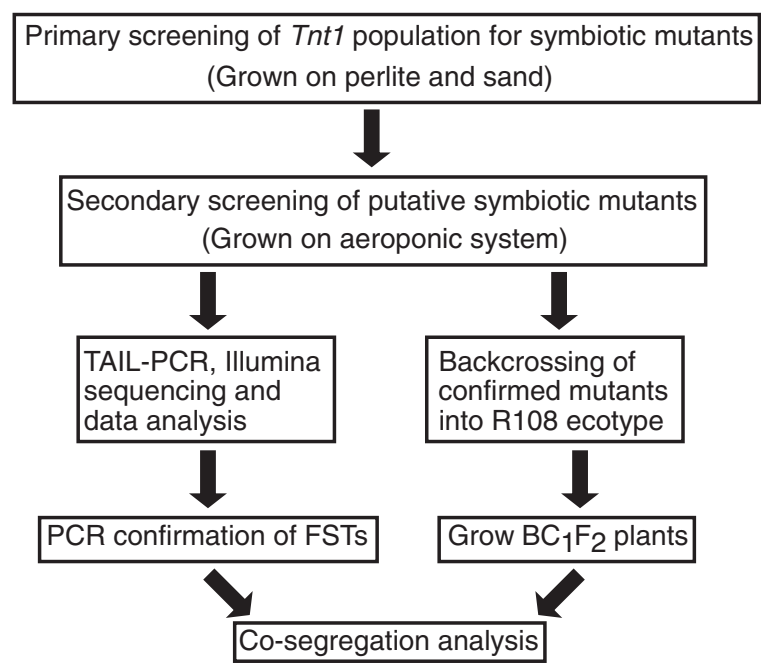

Fig. 1 Overview of forward genetic screening for symbiotic mutants and identification of causative genes. Steps in forward genetic screening and identification of causative genes responsible for $M$. truncatula Tnt1 mutants defective in nodule development and symbiotic nitrogen fixation are shown
Phenotypic characterization and segregation analysis of NF11217 and NF10547 mutants

Individual plants from NF11217 and NF10547 lines show reddish purple leaves and form small spherical, white ineffective Fix- nodules (Fig. 2a, b). X-Gal stained nodule sections show that NF11217 and NF10547 nodules failed to form distinct nodule zones and show reduced rhizobial occupancy (Fig. 2c). To study the inheritance and penetrance of Nod + Fix- phenotypes, we backcrossed the mutants into the parental R108 ecotype [23]. All the $\mathrm{BC}_{1} \mathrm{~F}_{1}$ plants from successful

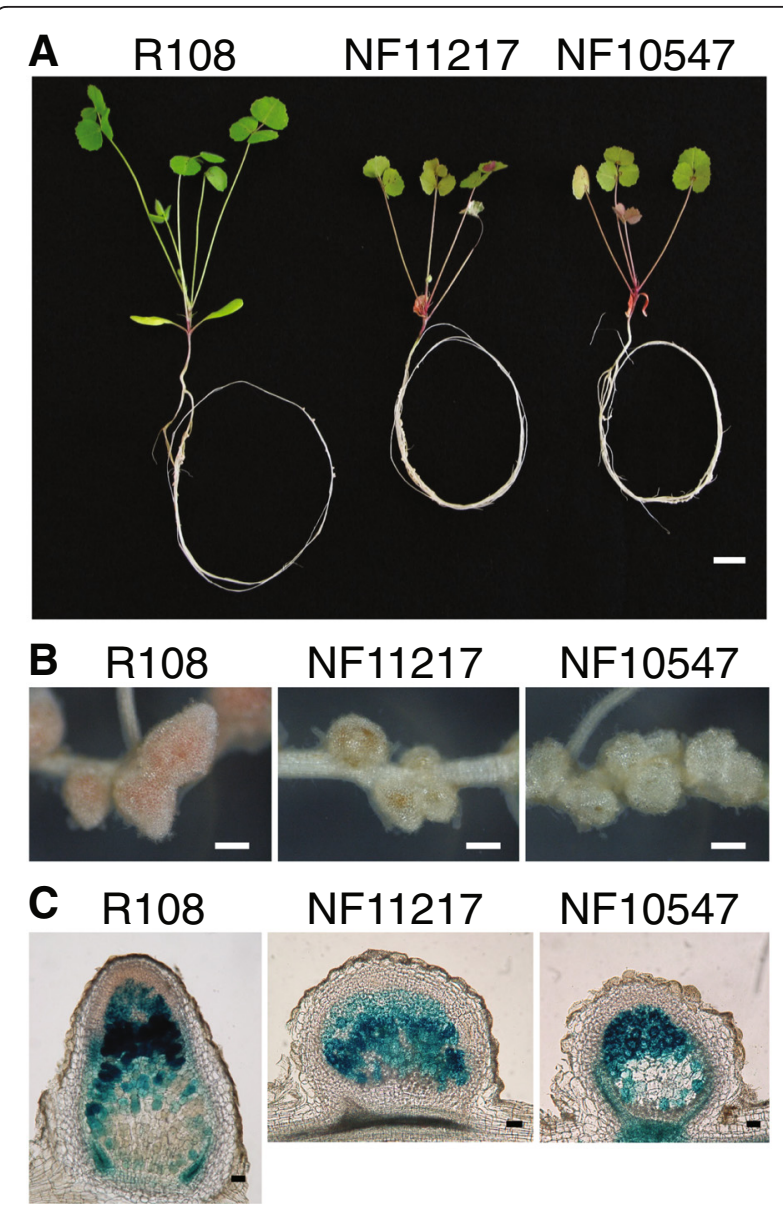

Fig. 2 Phenotypic characterization of NF11217 and NF10547 plants. Plants were grown on aeroponic chambers in the presence of $5 \mathrm{mM}$ ammonium nitrate for 5 days, followed by 5 days without nitrogen, and subsequently inoculated with S. meliloti Rm41 hemA:lacZ. Phenotyping was performed 15 days post inoculation. a Whole plants. WT leaves and petioles are green while the mutants' leaves and petioles are reddish purple, caused by anthocyanin accumulation during nitrogen deficiency. Scale bar $=1 \mathrm{~cm}$. b Visible nodule phenotype. WT nodules are pink; in comparison the mutants' nodules are brownish or white. Scale bar $=0.5 \mathrm{~mm}$. c Rhizobial occupancy nodule phenotype. The nodules were fixed and stained with X-Gal (5-bromo-4-chloro-3-indolyl-beta-D-galacto-pyranoside), sectioned to 100-micron-thickness, and imaged using bright field. scale bar $=100 \mu \mathrm{m}$ 
crosses showed wild-type shoot and nodule characteristics. The $\mathrm{BC}_{1} \mathrm{~F}_{1}$ plants were allowed to self-fertilize. Phenotyping of $\mathrm{BC}_{1} \mathrm{~F}_{2}$ plants showed a 3:1 (wild-type: mutant) segregation ratio for phenotypes associated with SNF defects (Table 1). These results indicate that the defective symbiotic phenotypes in NF10547 and NF11217 mutants are governed by monogenic, recessive mutations.

\section{Recovery of flanking sequence tags (FSTs) using TAIL-PCR} The $M$. truncatula Tnt1 mutant database contains 333,482 high confidence flanking sequence tags (FSTs) and 406,299 low confidence FSTs (http://medicagomutant.noble.org/mutant/database.php). These FSTs were recovered by TAIL-PCR from $\mathrm{R}_{0}$ hemizygote plants [5]. In an effort to identify the mutations responsible for the defective symbiotic phenotypes in the relevant mutants, we used the available FSTs to develop genetic markers to map and potentially identify the causative Tnt1 insertions. To identify the genes with Tnt1 inserts, we performed BLAST analysis of the FSTs using R108 BLAST web browser (http://www.medicagohapmap.org/ tools/r108_blastform) which aligns the FST genomic sequences to the R108 draft genome as well as the reference A17 genome [25, 26]. Because we anticipated that potential causative Tnt1 insertions might be in nodule-specific genes, we cross-referenced to the $M$. truncatula gene expression atlas (MtGEA) database (http://mtgea.noble.org/v3/; [27]) and the A17 genome browser (JBrowse Mtv4.0; http://jcvi.org/medicago/ browsers.php) for nodule gene expression.

For line NF11217, we found a total of 31 high confidence FSTs in the $M$. truncatula Tnt1 database (Additional file 1) that mapped to the A17 genome (Additional file 2: Table S1). In addition, we performed a second round of TAIL-PCR from a single NF11217 Nod + Fix- plant in the $\mathrm{R}_{1}$ generation and recovered a total of $39 \mathrm{FSTs}$ (Additional file 1). Among the 39 FSTs, 30 were new and not recovered from $R_{0}$ plants in the first round of TAIL-PCR. Among these $30 \mathrm{R}_{1}$ FSTs, 27 mapped to the A17 and R108 genomes, while two mapped only to the R108 genome, and one matched neither (Additional file 2: Table S1 and Table 2). Among the recovered 61 FSTs in total, none of
Table 2 Recovery of flanking sequence tags (FSTs) from Tht 1 insertion mutants NF11217 and NF10547 using TAIL-PCR from different generations

\begin{tabular}{llll}
\hline Tnt1 mutant line & Generation & Total FSTs & Novel FSTs $^{a}$ \\
\hline NF11217 & $R_{0}$ & 31 & 31 \\
& $R_{1}$ & 39 & 30 \\
& $B_{1} F_{2}$ & 18 & 10 \\
NF10547 & Total & 88 & 71 \\
& $R_{0}$ & 14 & 14 \\
& $R_{2}$ & 25 & 24 \\
& Total & 39 & 38 \\
\hline
\end{tabular}

IInsertion locations of FSTs in the A17 genome from $R_{0}$ versus $R_{1}$, and $R_{0}$ and $R_{1}$ versus $B C_{1} F_{2}$ and $R_{0}$ versus $R_{1}$ were compared for NF11217 and NF10547, respectively to identify novel, non-redundant FSTs

the annotated genes interrupted by Tht1 insertions were nodule-specific based on the MtGEA database and A17 genome browser. A third round of TAIL-PCR from two single $\mathrm{BC}_{1} \mathrm{~F}_{2}$ NF11217 Nod + Fix-mutant plants recovered 18 FSTs, with 10 novel FSTs compared to the previous two rounds. As a whole, we isolated 71 unique FSTs by TAILPCR in three different generations including $R_{0}, R_{1}$ and $\mathrm{BC}_{1} \mathrm{~F}_{2}$. Among them, 67 were mapped to unique sites in the A17 genome, 3 matched only to the R108 genome and there was one FST that did not match to either A17 or R108 genomes. None of the recovered FSTs were mapped to nodule-specific genes (Additional file 2: Table S1 and Table 2).

For the NF10547 mutant, we obtained 14 FSTs $\left(\mathrm{R}_{0}\right.$ generation) from Tnt1 mutant database (Additional file 3) and among them 10 FSTs were mapped to the A17 genome. None of these FSTs were found to be obvious candidate genes based on nodule specific gene expression (Additional file 4: Table S2). We performed an additional TAIL-PCR from an individual $\mathrm{R}_{2}$ mutant and recovered 24 novel FSTs. As noted below, the same individual $R_{2}$ mutant was subjected to WGS analysis. The TAIL-PCR analysis yielded a total of 38 non-redundant FSTs from NF10547 TAILPCR. Among them, only 34 FSTs were mapped to the A17 genome (Additional file 3; Additional file 4: Table S2 and Table 2).

Table 1 Segregation analyses of NF11217 and NF10547

\begin{tabular}{|c|c|c|c|c|c|}
\hline \multirow{2}{*}{$\begin{array}{l}\text { Cross } \\
\text { (Female } \times \text { Male) }\end{array}$} & \multirow[t]{2}{*}{ Generation } & \multirow{2}{*}{$\begin{array}{l}\text { Total } \\
\text { Plants }\end{array}$} & \multicolumn{2}{|l|}{ Phenotype } & \multirow[t]{2}{*}{$x 2^{a}$} \\
\hline & & & Wild-type (Pink nodule and green shoot) & Nod + Fix- mutant (White nodule and purple plant) & \\
\hline \multirow[t]{2}{*}{ NF11217 × R108 } & $F_{1}$ & 8 & 8 & 0 & - \\
\hline & $\mathrm{F}_{2}$ & 328 & 258 & 70 & 2.756 \\
\hline \multirow[t]{2}{*}{ NF10547 × R108 } & $F_{1}$ & 9 & 9 & 0 & - \\
\hline & $\mathrm{F}_{2}$ & 335 & 248 & 87 & 0.168 \\
\hline
\end{tabular}

${ }^{*} X^{2}$ analysis was calculated based on the expected 3:1 (wild-type: mutant) ratio, $P>0.05$ 
Whole genome sequencing to recover Tnt 1 insertion sites WGS approaches have been shown to be more sensitive and efficient, and have identified the causative T-DNA insertions in Arabidopsis thaliana and fungal mutants obtained from forward genetic screens in which TAIL-PCR, plasmid rescue and adapter ligation PCR techniques failed $[16,17,19]$. Hence, we attempted WGS approach on the M. truncatula mutants using the Illumina Hiseq 2000 platform that provided 188 and 180 million 90 bp pairedend (PE) clean reads from $500 \mathrm{bp}$ insert libraries representing 44X and 40X total genome coverages of NF11217 and NF10547 mutants, respectively (Table 3).

Paired end (PE) sequencing generated two 90 bp short reads from both ends of a DNA fragment hereafter referred to as read 1 (R1) and read 2 (R2) (Fig. 3a). Each read from $\mathrm{PE}$ sequencing could match completely to the reference A17 genome (genomic), or Tht1 sequences only (Tnt1) or it could be a hybrid sequence comprised of parts of Tht 1 element and R108 genome, thus representing the insertion site (hybrid). The PE reads (R1-R2) from WGS can be classified as follows based on the sequence composition: (i) Type 1: Tnt1-Tnt1, (ii) Type 2: genomic-genomic, (iii) Type 3: hybrid-Tnt1 or Tnt1-hybrid, (iv) Type 4: genomichybrid or hybrid-genomic and (v) Type 5: genomic-Tnt1 or Tnt1-genomic (Fig. 3a). Type 2 reads could be used to estimate the zygosity of insertion loci based on the genomic reads that mapped to the insertion junction sites [28]. The PE reads from Types 3, 4, and 5 are informative for the identification of the Tnt1 insertion sites.

An overview of the data analysis of WGS data to identify the Tnt1 insertion sites are outlined in Fig. 3b. To detect the Tnt1 insertion sites from the WGS data, we employed a stand-alone BLAST program [29] set up on a Linux platform to align reads against the Tnt1 sequence. This was done for all our BLAST analyses because the $M$. truncatula reference genome is from the A17 ecotype, while the Tnt1 mutants are in R108 background, and therefore, alignment against Tnt1 sequences alone circumvents the ambiguities because of

Table 3 Summary of whole genome sequencing data and coverage estimates

\begin{tabular}{lll}
\hline & \multicolumn{2}{l}{ Tnt1 mutant line } \\
\cline { 2 - 3 } & NF11217 & NF10547 \\
\hline Total number of raw reads (millions) & 196.31 & 184.11 \\
Total nucleotides of raw reads (Giga bases) & 17.67 & 16.57 \\
Total number of clean reads (millions) $^{\mathrm{a}}$ & 188.03 & 176.77 \\
Total nucleotides of clean reads (Giga bases) & 16.92 & 15.91 \\
Total genome coverage of clean reads $^{\mathrm{b}}$ & $44 \mathrm{X}$ & $41 \mathrm{X}$ \\
\hline
\end{tabular}

${ }^{a}$ After removing the adapter sequences and reads that contain $>50 \%$ low quality bases

${ }^{{ }^{6}}$ Total genome coverage was calculated based on the estimated Medicago truncatula A17 genome size of $384 \mathrm{Mb}$ Krishnakumar et al., [26] using the formula clean bases/reference genome size polymorphisms between A17 and R108. We aligned the PE reads $R 1$ and $R 2$ to both the left and right ends of Tnt1 and recovered Types 3, 4 and 5 reads (Fig. 3a). Type 4 and type 5 reads were assembled into contigs, called nodes. Subsequently, we aligned the hybrid reads and nodes against the A17 reference genome to identify the genomic co-ordinates of the Tht1 insertions. Ideally, each unique Tht1 insertion should be represented by all 6 types of supporting hybrid reads and nodes: Types 3,4 and 5 for each side of the Tnt1 insertion. Insertion loci with at least 3 supporting hybrid reads and/or nodes are considered high confidence $(\mathrm{HC})$ whereas the rest of the loci are considered low confidence (LC). From this analysis, we identified $97 \mathrm{HC}$ insertion loci for NF11217 with 89 mapped to the A17 reference genome and the remaining 8 mapped to the A17 scaffolds only (Additional file 5: Table S3 and Table 4). We also found 1078 LC insertion loci for NF11217 (Additional file 6: Table S4). For NF10547, we identified a total of $65 \mathrm{HC}$ insertion loci. Among the $\mathrm{HC}$ loci, 52 were mapped to specific chromosomal locations while the remaining loci mapped to A17 scaffolds (Additional file 7: Table S5 and Table 4). For NF10547, 268 LC Tnt1 loci were obtained (Additional file 8: Table S6). Mapping of some of these insertion loci showed that they are in the A17 scaffolds instead of chromosomal locations indicating gaps in the $M$. truncatula genome. The significance of the numerous LC insertion loci obtained for both lines is unclear.

We attempted comparison between the HC Tnt1 insertion locations obtained via TAIL-PCR and those obtained by WGS, although they are not strictly comparable because the TAIL-PCR Tnt1 insertion locations were obtained from different generations of segregating and back-crossed populations, while the WGS data was obtained from only an individual $\mathrm{BC}_{1} \mathrm{~F}_{2}$ (in the case of NF11217) or $\mathrm{R}_{2}$ (for NF10547) mutant plant. For NF11217, TAIL-PCR identified a total of 71 Tnt1 insertions while WGS recovered 97 HC insertion loci, with 27 Tnt1 insertion loci identified by both approaches (Additional file 9: Table S7 and Fig. 4a). For NF10547, 38 unique TAIL-PCR and 65 HC WGS Tnt1 FSTs were found with 11 FSTs common to both datasets (Additional file 10: Table S8 and Fig. 4b). These data indicate that the WGS approach identified substantially more Tht1 insertion locations than TAIL-PCR. Additionally, the Tnt 1 insertion locations identified by WGS were from an individual $\mathrm{BC}_{1} \mathrm{~F}_{2}$ or $\mathrm{R}_{2}$ mutant plant for NF11217 and NF10547 respectively, which have already lost some non-co-segregating Tnt1 insertions present in the $\mathrm{R}_{0}$ and $\mathrm{R}_{1}$ generations that were subjected to TAIL-PCR.

NF11217 and NF10547 mutants are novel Tnt 1 insertion alleles of PLC-like and NIN genes, respectively

To identify the causative Tnt1 insertions underlying the nodulation defects in NF11217 and NF10547, we 


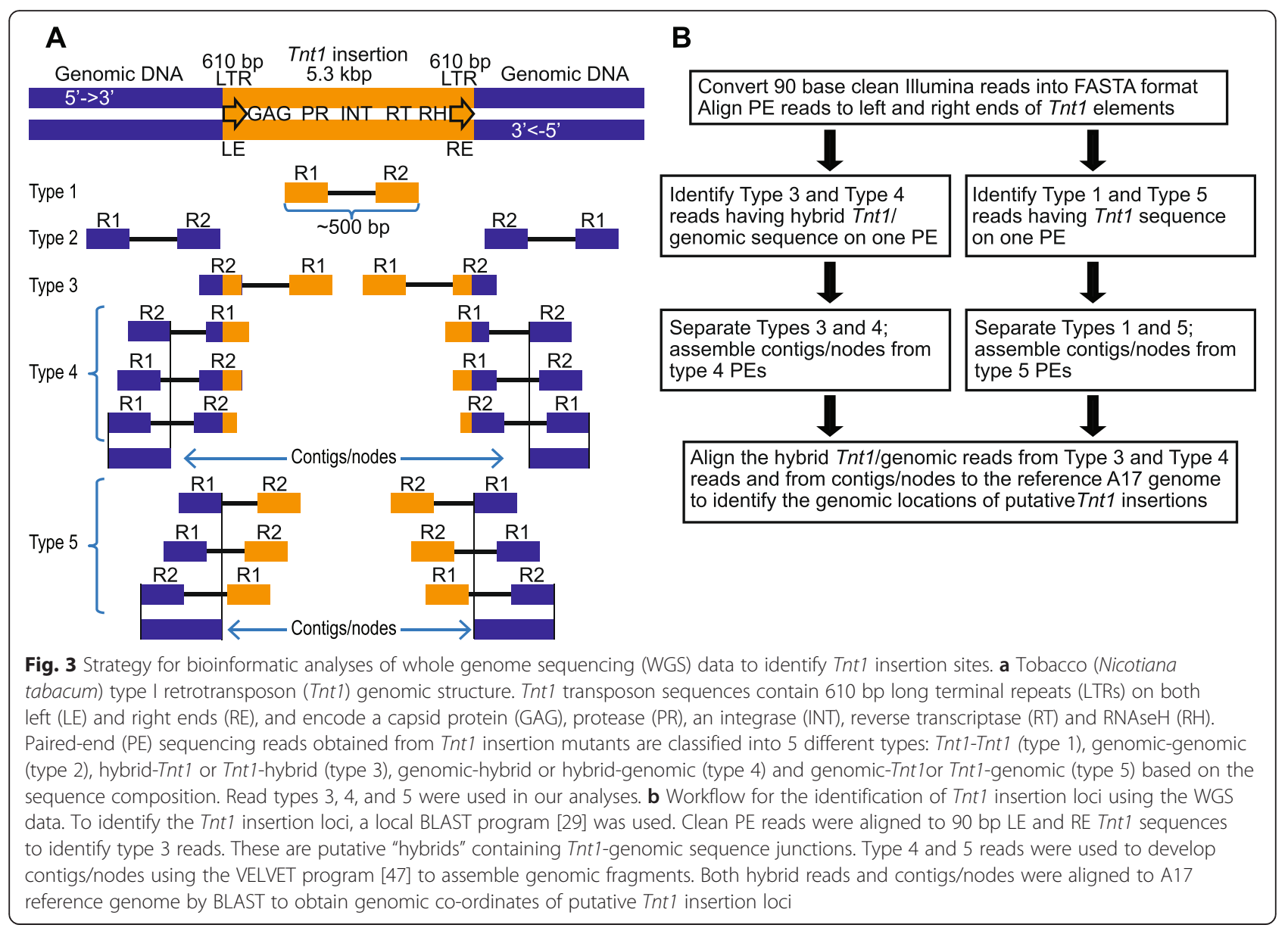

analyzed the genome coordinates of insertion loci from WGS data. One of the Tnt1 insertion loci, Insertion-38, in NF11217 with 69 supporting reads/nodes (Additional file 5: Table S3; Additional file 9: Table S7) mapped to the previously characterized DEFECTIVE IN NITROGEN FIXATION 2 (DNF2) gene encoding a PLC-like family protein (Medtr4g085800). BLAST analysis revealed this Tnt1 insertion maps to the $6^{\text {th }}$ exon of DNF2/PLC-like (Fig. 5a). The PLC-like protein was reported to play an essential role in nodule development and symbiotic nitrogen fixation under non-permissive conditions [15, 30-32]. Mutant NF11217 plants have similar phenotypes (Fig. 2) to those of the previously characterized $d n f 2$ mutant NF0217 in our growth conditions (Additional file 11: Figure S1). Some $d n f 2$ mutant nodules have an apparent defense-like reaction producing brown-colored phenolic compounds, while others are white (Fig. 2b, c; Additional file 11: Figure S1), like the phenotypes described when plants are grown on media solidified with agar [15]. The reasons for the variation in nodule phenotype in $d n f 2$ mutant nodules is unknown [15, 32]. Genetic markers for the defective Medtr4g085800 allele were made and found to cosegregate with the defective SNF phenotype in the $\mathrm{BC}_{1} \mathrm{~F}_{2}$ population, with all WT $\mathrm{BC}_{1} \mathrm{~F}_{2}$ plants in the population carrying at least one WT allele of Medtr4g085800 (Fig. 5a; Additional file 12: Table S9). Hence, NF11217 is a new allele of $d n f 2$, which we call dnf2-5.

Analysis of WGS data for NF10547 identified a Tnt1 insertion in the fourth exon of a previously characterized nodule specific gene NODULE INCEPTION (NIN; Medtr5g099060). This insertion locus, Insertion-31, has 54 supporting reads/nodes (Additional file 7: Table S5;

Table 4 High confidence Tnt1 insertion site statistics from whole genome sequencing

\begin{tabular}{llll}
\hline Tnt1 & Total Tnt1 & Tnt1 insertion loci & Mapped to A17 scaffolds \\
\cline { 2 - 4 } mutant line & insertion loci & 89 & 8 \\
NF11217 & 97 & 52 & 13 \\
NF10547 & 65 & Mapped to A17 chromosomes & 13 \\
\hline
\end{tabular}




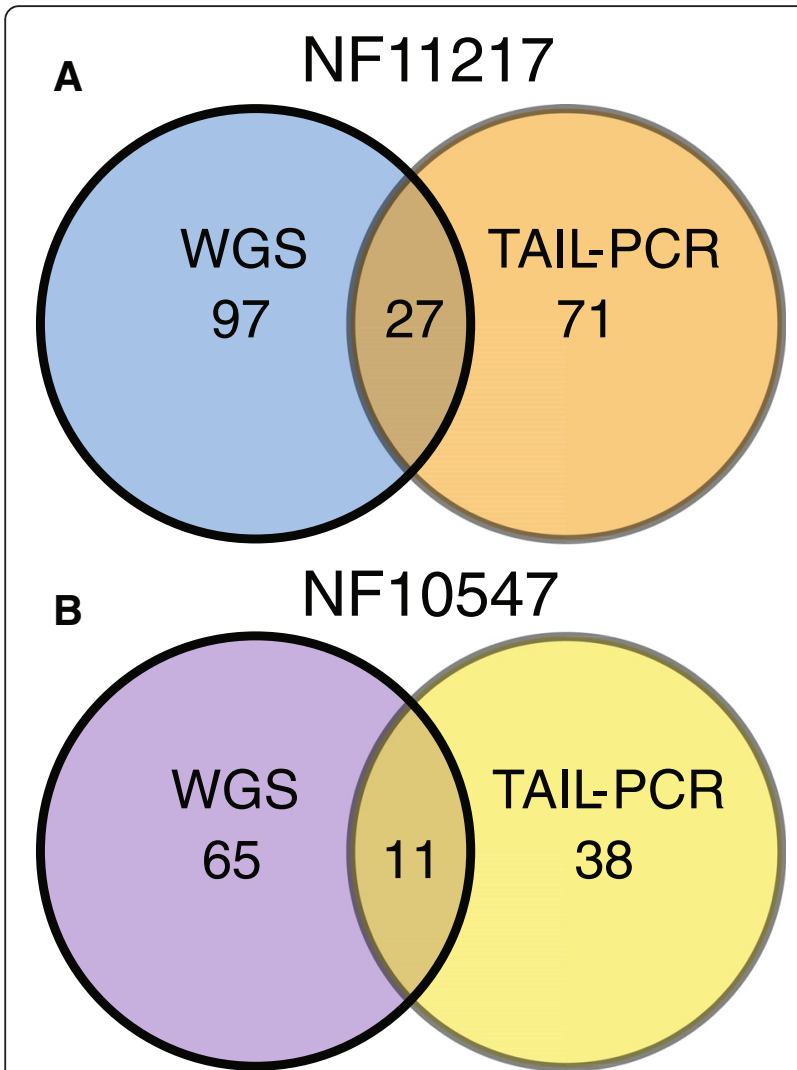

Fig. 4 Comparison of Tht 1 insertions obtained from TAIL-PCR and WGS. a Unique high confidence Tnt1 insertion sites that were recovered from $R_{0}, R_{1}$ and $B C_{1} F_{2}$ generations of NF11217 by TAIL-PCR were compared to those obtained from a single $\mathrm{BC}_{1} \mathrm{~F}_{2}$ plant by WGS. b Unique high confidence Tnt1 insertion sites that were obtained from $R_{0}$ and $R_{2}$ generations of NF10547 were compared to those obtained by WGS from a single $R_{2}$ plant

Additional file 10: Table S8) confirming the Tnt1 insertion in NIN gene. NIN is a key transcriptional factor which plays a major role in the formation of infection threads, induction of cortical cell divisions and regulation of nodule formation [33-38]. In this case, the second round of TAIL-PCR obtained from a single NF10547 Nod + Fix- plant from $R_{2}$ generation also identified a Tnt1 insertion in NIN (Additional file 4: Table S2). To test whether the interrupted NIN gene is responsible for the underlying Nod + Fix- phenotype of NF10547 Tnt1 mutant line, we performed cosegregation analysis using the $\mathrm{BC}_{1} \mathrm{~F}_{2}$ population obtained from a NF10547 $\times$ R108 cross. PCR genotyping showed that all tested $\mathrm{BC}_{1} \mathrm{~F}_{2}$ plants with a Nod + Fixphenotype were homozygous for the Tnt1 insertion in NIN gene whereas all the tested WT phenotype plants had at least one WT uninterrupted NIN allele (Fig. 5b and Additional file 13: Table S10). These data indicate that NF10547 contains a new nin allele, which we have named nin-16. Most of the previously reported $M$.

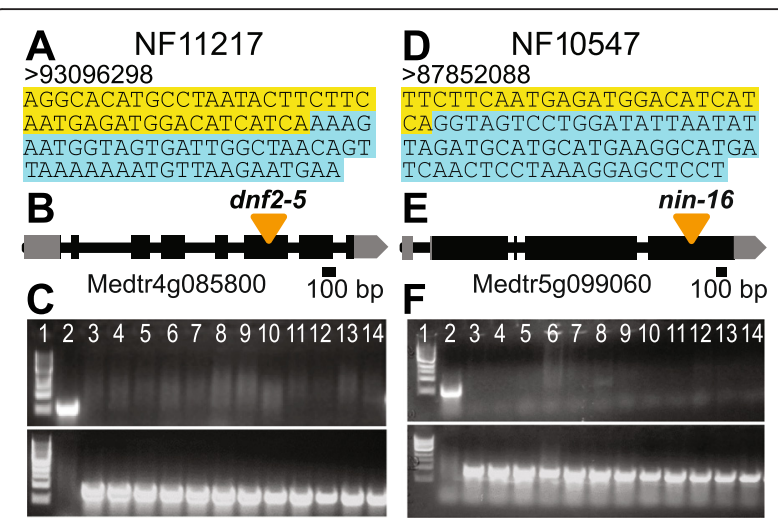

Fig. 5 NF11217 and NF10547 contain novel Tht1 insertions in NODULE INCEPTION (NIN) and PHOSPHOLIPASE C (PLC)-like, respectively. a A hybrid read example obtained for NF11217 with the causative Tnt1 insertion showing the Tnt1 border sequence (yellow) and PLC-like sequence (blue). $\mathbf{b}$ Genomic structure of PLC-like indicating the Tnt1 insertion site in NF11217. c Representative co-segregation data for Tnt1 insertions in NF11217 co-segregating with Nod + Fix- plants. The top panel shows PCR products from PCR reactions with two primers specific for PLC-like genomic sequences, Medtr4g085800-1 F and Medtr4g085800-1R, of 585 bp; the lower panel shows PCR products with one PLC-like genomic primer, Medtr4g085800-1 F, and one Tnt1 primer, Tnt-F1, of 805 bp. Lane 1 shows DNA markers (New England Biolabs, Beverly, MA; \#N3232); lane 2, WT R108 DNA; and lanes 3-14, Nod + Fix- plants in the NF11217 $B C_{1} F_{2}$ population. d A hybrid read example obtained for NF10547 with the causative Tnt1 insertion showing the Tnt1 border sequence (yellow) and NIN sequence (blue). e Genomic structure of NIN indicating the Tnt 1 insertion site in NF10547. f Representative co-segregation data for Tnt1 insertions in NF10547 co-segregating with Nod + Fix- plants. The top panel shows PCR products from $P C R$ reactions with two primers specific for NIN genomic sequences, Medtr5g099060-1 F and Medtr5g099060-1R, of 503 bp; the lower panel shows PCR products with one NIN genomic primer, Medtr5g099060-1 F, and one Tnt1 primer, Tnt-R1, of 810 bp. Lane 1 shows DNA markers; lane 2, WT R108 DNA; and lanes 3-14, $\mathrm{Nod}+$ Fix- plants in the NF10547 $\mathrm{BC}_{1} \mathrm{~F}_{2}$ population. Additional PCR genotyping data for NF11217 and NF10547 WT-like and Nod + Fix- plants from $\mathrm{BC}_{1} \mathrm{~F}_{2}$ can be found in Additional file 12: Table 59 and Additional file 13: Table S10, respectively. Genotyping primers are listed in Additional file 15: Table S11

truncatula nin mutants showed a Nod- phenotype [13, 34]. Conceptual translation of nin-16 shows that it encodes a putative 795 amino acid hybrid protein, with the first 785 amino acid residues of NIN followed by ten amino acid residues encoded by Tnt1 (Additional file 14: Figure S2). It has the first six conserved blocks of sequence found in NIN and NIN-like proteins, including the characteristic RWP-RKP domain thought to be important for dimerization and DNA binding activity in regulation of genes controlled by nitrogen status [33, 39], but lacks the last conserved sequence block containing the PB1 domain, putatively vital for hetero-dimerization [39, 40] (Additional file 14: Figure S2). Our genetic data lead us to hypothesize that the Fix-nodule phenotype in NF10547 is likely caused by the weak nin-16 allele as 
opposed to a cryptic defect in a different, closely-linked gene. Other observations support the idea that a weak nin allele could have such a phenotype. First, there is a brief description of another M. truncatula nin allele, also a Tnt1 insertion in the last exon of NIN, as Nod+/Fix-, while other nin alleles with Tnt1 insertions in the last NIN exon are Nod- [13]. Second, in mature determinate L. japonicus nodules, NIN expression persists which indicates that NIN likely has a role later in nodule development as well as in nodule inception [33]. Third, NIN expression in indeterminate $M$. truncatula nodules has highest expression in the nodule infection zone and significant expression in the inter-zone and nitrogen fixing zones [41]. Our data indicate that NF10547 is expected to contain a partially functional NIN protein. This new allele could be valuable in further dissecting NIN's function after nodule inception, in rhizobial infection and in nodule organogenesis.

Overall, our data show that while TAIL-PCR is useful in identifying the causative Tht1 FSTs in some mutants, WGS is more efficient in pinpointing the FSTs. In this work, with $>40 \mathrm{X}$ total genome coverage, we were able to identify the mutations underlying the defects in two mutants. Previously, it was reported that 10X genome coverage is enough to identify $96 \%$ of the insertions from M. truncatula [28]. The optimal sequencing coverage required to identify the causative insertions in mutants from a forward genetic screen was not reported previously for M. truncatula. WGS is increasingly becoming more cost-effective and accurate. The availability of several commercial vendors for genome sequencing and bioinformatics analysis has facilitated rapid data acquisition, allowing researchers to focus on the genetic characterization of identified genes and biochemical characterization of their encoded proteins, speeding up forward genetics. TAIL-PCR is still a valuable tool for forward genetics, but the success rate of WGS for forward genetic screens is significantly higher, as reported in previous studies in other organisms $[16,17,19]$, and now established for M. truncatula by our study.

\section{Conclusions}

In this work, we demonstrate that WGS is an efficient approach for the recovery of high numbers of Tnt1 insertion sites from $M$. truncatula Tnt1 insertion mutants. Our results demonstrated that WGS efficiency clearly surpassed that of TAIL-PCR. We also showed the utility of the WGS method in identifying relevant disrupted genes in two mutants isolated in a forward genetic screen as defective in SNF nodule development. This work uncovered a new $d n f 2-5$ allele with phenotypes similar to $d n f 2$ mutants previously described. It also described a new nin-16 mutant that showed defects later in nodule development than other nin mutants. This weak nin allele is likely to be valuable in characterizing NIN's functions later in nodule development, after nodule inception.

\section{Methods \\ Plant materials, growth conditions and genetic crossing}

$M$. truncatula plant growth conditions and genetic crossing procedures were as described [23].

\section{Nodule phenotyping, fixing of nodules, sectioning and X-Gal staining}

Primary mutant screening was performed using Tnt 1 mutant population grown under low nitrate conditions $\left(0.5 \mathrm{mM} \mathrm{KNO}_{3}\right)$ on a mixture of perlite and sand $(3: 1)$ inoculated with a rhizobial strain S. meliloti Sm1021 as described in Yarce et al. [20]. Four weeks after inoculation, plants were uprooted and screened for visible defective symbiotic phenotypes. For secondary screening, putative mutants were grown on aeroponic chambers containing plant growth media [42] supplemented with $5 \mathrm{mM} \mathrm{NH}_{4} \mathrm{NO}_{3}$ for 5 days followed by 5 days of growth without any nitrogen source. Subsequently, plants were inoculated with S. meliloti Rm41 carrying the hemA:lacZ reporter (gift from Dr. Pascal Ratet). Nodules were fixed and stained with X-Gal as described previously [43]. Nodule sections, $100 \mu \mathrm{m}$ thick were obtained using a 1000 Plus model Vibratome (Vibratome, St. Louis, MO). Sections were observed and documented under an Olympus BX50 microscope using bright field settings.

\section{Preparation of genomic DNA and PCR genotyping}

Genomic DNA (gDNA) from M. truncatula was prepared from mature leaves. gDNA for PCR genotyping and TAIL-PCR was prepared using an established method [44] and gDNA for whole genome sequencing was prepared using a modified CTAB method as described (www.monsanto.com/products/documents/dnadetection/dna_im.pdf). Genotyping primers used for cosegregation analysis were designed using R108 and A17 genomic sequences downloaded from the R018 BLAST browser from $M$. truncatula Hapmap website (http://www.medicagohapmap.org/tools/r108_blastform). PCR was performed in $20 \mu \mathrm{l}$ reactions using Go-Taq Green Master Mix (Promega, Madison, WI; Cat. No. M7123). Primers sequences for genotyping and co-segregation analysis are listed in Additional file 15: Table S11.

\section{TAIL-PCR}

Thermal asymmetric interlaced (TAIL)-PCR was performed as described $[5,45,46]$. For the primary PCR amplification, Tnt1-specific primers Tnt1-F (forward) or Tnt1-R (reverse) in combination with five different arbitrary degenerate $(\mathrm{AD})$ primers $\mathrm{AD} 1, \mathrm{AD} 2, \mathrm{AD} 3, \mathrm{AD} 5$ and $\mathrm{AD} 6$ were used for each Tnt1 individual mutant genomic DNA template. The 50-fold-diluted primary PCR products were used as 
templates for the secondary PCR. For the $2^{\text {nd }}$ PCR amplification, a nested Tnt1-specific primer which is close to the end of the Tnt1 (Tnt1-F1 or Tnt1-R1) was used for each individual template in combination with the same five $\mathrm{AD}$ primers that were used in the primary PCR amplification. After the $2^{\text {nd }} \mathrm{PCR}$ amplification, PCR products were purified by Qiagen PCR Purification Kit (Qiagen, Valencia, CA; Cat. No. 28104), quantified by NanoDrop Spectrophotometer (Thermo Scientific, Wilmington, DE) and ligated to pGEM-T-Easy vector system (Promega, Madison, WI; Cat. No. A1360). Plasmids from 96 random white colonies were sequenced using Sanger sequencing from each side of the Tnt 1 for each mutant lines. Primers used in TAIL-PCR are listed in Additional file 15: Table S11.

\section{Library preparation and whole genome sequencing}

Library preparation, whole genome sequencing and data analysis to obtain clean reads were performed by the Beijing Genomics Institute (BGI), China (http://bgiinternational.com/us/?id=). Ninety bp PE reads were obtained from $500 \mathrm{bp}$ insert libraries by sequencing on an Illumina HiSeq 2000 platform.

\section{Bioinformatics analysis}

Alignments of PE reads to Tht1 sequences and the reference genome A17 were performed using BLAST programs [29] installed on a Linux machine. The BLAST output was parsed using a custom Perl script to identify the hybrid reads containing Tnt1-genomic junction sequences, as outlined in Fig. 3. Genomic reads obtained from PE sequences in which the other reads are either Tnt1 or hybrid, were further developed into contigs/ nodes using the VELVET program [47]. Hybrid reads and genomic contigs were aligned to the $M$. truncatula reference genome A17 using BLAST to identify the genome co-ordinates of putative Tht1 insertion sites. A Perl script was developed to cluster the hybrid reads and the VELVET-derived contigs from both the hybrid read paired-ends and the Tnt1 paired-ends. The grouping of the reads was based on alignment to the A17 genome using BLAST. Each group or cluster (putative insertion) thus obtained was assigned a confidence level of either high or low, based on the number of supporting reads/ nodes. Clusters derived from 3 or more reads/nodes were considered to be high confidence, otherwise they were annotated as low confidence. Clusters were sorted by their confidence level, then by chromosome number and position on the chromosome, and finally given unique insertion numbers based on this ordering.

\section{Availability of supporting data}

WGS data that was used in identifying the Tnt1 insertion sites were deposited in the NCBI Sequence Read
Archive (SRA) (http://www.ncbi.nlm.nih.gov/sra) under the BioProject accession number PRJNA298564 with experiment accession numbers SRR2650316 (NF11217) and SRX1335995 (NF10547).

\section{Additional files}

Additional file 1: List of sequences of all NF11217 flanking sequence tags (FSTs) obtained from $R_{0}, R_{1}$ and $B C_{1} F_{2}$ generations by TAlL-PCR. (PDF $62 \mathrm{~kb}$ )

Additional file 2: Table S1. Chromosomal locations of NF11217 TAlL-PCR FSTs recovered from $R_{0}, R_{1}$ and $B C_{1} F_{2}$ generations. (XLS $53 \mathrm{~kb}$ )

Additional file 3: List of sequences of all NF10547 flanking sequence tags (FSTs) obtained from $R_{0}$ and $R_{2}$ generations by TAIL-PCR. (PDF $42 \mathrm{~kb}$ )

Additional file 4: Table S2. Chromosomal locations of NF10547 TAIL-PCR FSTs recovered from $R_{0}$ and $R_{2}$ generations. (XLS $43 \mathrm{~kb}$ )

Additional file 5: Table S3. List of high confidence Tnt1 insertion loci and its supporting reads/nodes identified from NF11217 by WGS. (XLS $509 \mathrm{~kb}$ )

Additional file 6: Table S4. List of low confidence Tnt1 insertion loci and its supporting reads/nodes identified from NF11217 by WGS. (XLS $345 \mathrm{~kb}$ )

Additional file 7: Table S5. List of high confidence Tnt1 insertion loci and its supporting reads/nodes identified from NF10547 by WGS. (XLS $294 \mathrm{~kb}$ )

Additional file 8: Table S6. List of low confidence Tnt1 insertion loci and its supporting reads/nodes identified from NF10547 by WGS. (XLS $141 \mathrm{~kb}$ )

Additional file 9: Table S7. Comparison of NF11217 high confidence Tht1 insertion loci identified from WGS vs TAIL-PCR. (XLS $48 \mathrm{~kb}$ )

Additional file 10: Table S8. Comparison of NF10547 high confidence Tnt1 insertion loci identified from WGS vs TAlL-PCR. (XLS $33 \mathrm{~kb}$ )

Additional file 11: Figure S1. Comparison between nodule phenotypes of NF11217 (dnf2-5), NF0217 (dnf2-2) and R108 (WT). (PDF $673 \mathrm{~kb}$ )

Additional file 12: Table S9. Co-segregation analysis of NF11217 Nod + Fix- phenotype with Tht1 insertion in PLC-like gene. (PDF 41 kb)

Additional file 13: Table S10. Co-segregation analysis of NF10547 Nod + Fix- phenotype with Tnt1 insertion in NIN gene. (PDF 42 kb)

Additional file 14: Figure S2. Comparison of Mtnin-16's encoded sequence with that of Lotus japonicus NIN and M. truncatula NIN. (PDF 797 kb)

Additional file 15: Table S11. Sequences of primers used in this study. (PDF $7 \mathrm{~kb}$ )

\section{Abbreviations}

AD: Arbitrary degenerate; BC: Back cross; BLAST: Basic local alignment search tool; DNA: Deoxyribonucleic acid; EMS: Ethyl methane sulfonate; FNB: Fast neutron bombardment; gDNA: Genomic DNA; HC: High confidence; INT: Integrase; LacZ: $\beta$-galactosidase; LC: Low confidence; LTR: Long-terminal repeat; M. truncatula: Medicago truncatula; MtGEA: Medicago truncatula gene expression atlas; NIN: NODULE INCEPTION; Nod: Nodulation; PCR: Polymerase chain reaction; PE: Paired-end; PLC: PHOSPHOLIPASE C; PR: Protease;

$R_{0}$ : Regeneration 0 ; R1: Read 1; RNA: Ribonucleic acid; RNase H: Ribonuclease H; RT: Reverse transcriptase; SNF: Symbiotic nitrogen fixation; TAILPCR: Thermal asymmetric interlaced (TAIL)-PCR; T-DNA: Transfer-DNA; Tnt1: Transposable element of Nicotiana tabacum; WGS: Whole genome sequencing; WT: Wild-type; X-Gal: 5-Bromo-4-chloro-3-indolyl-Pgalactopyranoside.

Competing interests

The authors declare that they have no competing interests. 


\section{Authors' contributions}

$W$ and RD designed the experiments. MJ and TT performed bioinformatic analysis of WGS data. KK, RG, TM and ES performed co-segregation analysis. JW performed TAIL-PCR to obtain FSTs. W, KSM, RA and RD coordinated the experiments, and wrote and edited the manuscript. RG and TM are undergraduate co-authors. All authors read and approved the final manuscript.

\section{Acknowledgements}

We thank Pascal Ratet for the S. meliloti strain Rm41 harboring the hemA:lacZ fusion and members of the S.R. Noble Foundation greenhouse staff for their cheerful help during the $M$. truncatula community genetic screens. We thank Catalina Pislariu for sharing her images documenting the phenotype of the dnf2-2 mutant in our growth conditions, as shown in Additional file 11: Figure S1. This work was supported by National Science Foundation grant IOS-1127155 to RD and KSM.

\section{Author details}

'Department of Biological Sciences, University of North Texas, 1155 Union Circle \#305220, Denton, TX 76203, USA. ${ }^{2}$ Plant Biology Division, The Samuel Roberts Noble Foundation, Ardmore, OK 73401, USA. ${ }^{3}$ Department of Mathematics, University of North Texas, Denton, TX 76203, USA

Received: 27 October 2015 Accepted: 9 February 2016

Published online: 27 February 2016

\section{References}

1. Jones KM, Kobayashi H, Davies BW, Taga ME, Walker GC. How rhizobia symbionts invade plants: the Sinorhizobium-Medicago model. Nat Rev Microbiol. 2007:5:619-33.

2. Oldroyd GED, Murray JD, Poole PS, Downie JA. The rules of engagement in the legume-rhizobial symbiosis. Annu Rev Genet. 2011;45:119-44.

3. Cook DR. Medicago truncatula - a model in the making! Curr Opin Plant Biol. 1999;2:301-4.

4. Young ND, Udvardi M. Translating Medicago truncatula genomics to crop legumes. Curr Opin Plant Biol. 2009;12:193-201.

5. Tadege M, Wen J, He J, Tu H, Kwak Y, Eschstruth A, et al. Large-scale insertional mutagenesis using the Tnt1 retrotransposon in the model legume Medicago truncatula. Plant J. 2008:54:335-47.

6. Cheng $\mathrm{X}$, Wang $\mathrm{M}$, Lee $\mathrm{H}-\mathrm{K}$, Tadege $\mathrm{M}$, Ratet $\mathrm{P}$, Udvardi $\mathrm{M}$, et al. An efficient reverse genetics platform in the model legume Medicago truncatula. New Phytol. 2014;201:1065-76.

7. Grandbastien M-A, Spielmann A, Caboche M. Tnt1, a mobile retroviral-like transposable element of tobacco isolated by plant cell genetics. Nature. 1989:337:376-80.

8. Paszkowski J. Controlled activation of retrotransposition for plant breeding Curr Opin Biotechnol. 2015:32:200-6.

9. d'Erfurth I, Cosson V, Eschstruth A, Lucas H, Kondorosi A, Ratet P. Efficient transposition of the Tnt1 tobacco retrotransposon in the model legume Medicago truncatula. Plant J. 2003;34:95-106.

10. Mazier M, Botton E, Flamain F, Bouchet J-P, Courtial B, Chupeau M-C, et al. Successful gene tagging in lettuce using the Tnt1 retrotransposon from tobacco. Plant Physiol. 2007;144:18-31.

11. Cui Y, Barampuram S, Stacey MG, Hancock CN, Findley S, Mathieu M, et al. Tht1 retrotransposon mutagenesis: A tool for soybean functional genomics. Plant Physiol. 2013;161:36-47

12. Duangpan $\mathrm{S}$, Zhang $\mathrm{W}, \mathrm{Wu} Y$, Jansky SH, Jiang J. Insertional mutagenesis using Tnt1 retrotransposon in potato. Plant Physiol. 2013;163:21-9.

13. Pislariu Cl, Murray JD, Wen J, Cosson V, Muni RRD, Wang M, et al. A Medicago truncatula tobacco retrotransposon insertion mutant collection with defects in nodule development and symbiotic nitrogen fixation. Plant Physiol. 2012;159:1686-99.

14. Couzigou J-M, Zhukov V, Mondy S, Abu el Heba G, Cosson V, Ellis THN, et al. NODULE ROOT and COCHLEATA maintain nodule development and are legume orthologs of Arabidopsis BLADE-ON-PETIOLE genes. Plant Cell. 2012;24:4498-510.

15. Bourcy M, Brocard L, Pislariu Cl, Cosson V, Mergaert P, Tadege M, et al. Medicago truncatula DNF2 is a PI-PLC-XD-containing protein required for bacteroid persistence and prevention of nodule early senescence and defense-like reactions. New Phytol. 2013;197:1250-61.
16. Polko JK, Temanni M-R, van Zanten M, van Workum W, Iburg S, Pierik R, et al. Illumina sequencing technology as a method of identifying T-DNA insertion loci in activation-tagged Arabidopsis thaliana plants. Mol Plant. 2012;5:948-50.

17. Chambers K, Lowe RG, Howlett BJ, Zander M, Batley J, Van de Wouw AP et al. Next-generation genome sequencing can be used to rapidly characterise sequences flanking T-DNA insertions in random insertional mutants of Leptosphaeria maculans. Fungal Biol Biotechnol 2014; doi:10.1186/s40694-014-0010-y

18. Schneeberger K. Using next-generation sequencing to isolate mutant genes from forward genetic screens. Nat Rev Genet. 2014;15:662-76.

19. Wilson-Sánchez D, Rubio-Díaz S, Muñoz-Viana R, Pérez-Pérez JM, Jover-Gil S, Ponce MR, et al. Leaf phenomics: a systematic reverse genetic screen for Arabidopsis leaf mutants. Plant J. 2014;79:878-91.

20. Yarce JCS, Lee H-K, Tadege M, Ratet P, Mysore KS. Forward genetics screening of Medicago truncatula Tnt1 insertion lines. Methods Mol Biol. 2013:1069:93-100

21. Ott T, van Dongen JT, Gunther C, Krusell L, Desbrosses G, Vigeolas H, et al. Symbiotic leghemoglobins are crucial for nitrogen fixation in legume root nodules but not for general plant growth and development. Curr Biol. 2005;15:531-5.

22. Journet E-P, de Carvalho-Niebel F, Andriankaja A, Huguet T, Barker DG. Rhizobial inoculation and nodulation of Medicago truncatula. In: Mathesius U, Journet E-P, Sumner LW, editors. The Medicago truncatula Handbook. 2006. http://www.noble.org/MedicagoHandbook/: ISBN 0-9754303-1-9.

23. Veerappan V, Kadel K, Alexis N, Scott A, Kryvoruchk I, Sinharoy S et al. Keel petal incision: A simple and efficient technique for genetic crossing in Medicago truncatula. Plant Methods 2014; doi:10.1186/746-4811-10-11.

24. Hoffmann B, Trinh TH, Leung J, Kondorosi A, Kondorosi E. A New Medicago truncatula line with superior in vitro regeneration, transformation, and symbiotic properties isolated through cell culture selection. Mol Plant Microbe Interact. 1997;10:307-15.

25. Tang H, Krishnakumar V, Bidwell S, Rosen B, Chan A, Zhou S, et al. An improved genome release (version Mt4.0) for the model legume Medicago truncatula. BMC Genomics. 2014;15:312.

26. Krishnakumar V, Maria Kim, Rosen BD, Karamycheva S, Bidwell SL, Tang H et al. MTGD: The Medicago truncatula genome database. Plant Cell Physiol 2015; doi: 10.1093/pcp/pcu179.

27. Benedito VA, Torres-Jerez I, Murray JD, Andriankaja A, Allen S, Kakar K, et al. A gene expression atlas of the model legume Medicago truncatula. Plant J. 2008:55:504-13.

28. Jiang C, Chen C, Huang Z, Liu R, Verdier J. ITIS, a bioinformatics tool for accurate identification of transposon insertion sites using next-generation sequencing data. BMC Bioinformatics 2015; doi:10.1186/s12859-015-0507-2.

29. Altschul SF, Gish W, Miller W, Meyers EW, Lipman DJ. Basic Local Alignment Search Tool. J Mol Biol. 1990;215:403-10.

30. Starker CG, Parra-Colmenares AL, Smith L, Mitra RM, Long SR. Nitrogen fixation mutants of Medicago truncatula fail to support plant and bacterial symbiotic gene expression. Plant Physiol. 2006;140:671-80.

31. Berrabah F, Ratet $P$, Gourion B. Multiple steps control immunity during the intracellular accommodation of rhizobia. J Exp Bot. 2015;66:1977-85.

32. Berrabah F, Bourcy M, Cayrel A, Eschstruth A, Mondy S, Ratet P et al. Growth conditions determine the DNF2 requirement for symbiosis. PLoS One 2014; doi:10.1371/journal.pone.0091866.

33. Schauser L, Roussis A, Stiller J, Stougaard J. A plant regulator controlling development of symbiotic root nodules. Nature. 1999;402:191-5.

34. Marsh JF, Rakocevic A, Mitra RM, Brocard L, Sun J, Eschstruth A, et al. Medicago truncatula NIN is essential for rhizobial-independent nodule organogenesis induced by autoactive calcium/calmodulin-dependent protein kinase. Plant Physiol. 2007;144:324-35.

35. Soyano T, Kouchi H, Hirota A, Hayashi M. NODULE INCEPTION directly targets NF-Y subunit genes to regulate essential processes of root nodule development in Lotus japonicus. PLoS Genetics 2013; doi:10.1371/journal. pgen.

36. Soyano T, Hirakaw H, Sato S, Hayashi M, Kawaguchi M. NODULE INCEPTION creates a long-distance negative feedback loop involved in homeostatic regulation of nodule organ production. Proc Natl Acad Sci U S A. 2014;111:14607-12.

37. Soyano T, Shimoda Y, Hayashi M. NODULE INCEPTION antagonistically regulates gene expression with nitrate in Lotus japonicus. Plant Cell Physiol. 2015;56:368-76 
38. Yoro E, Suzaki T, Toyokura K, Miyazawa H, Fukaki H, Kawaguchi M. A Positive Regulator of Nodule Organogenesis, NODULE INCEPTION, Acts as a Negative Regulator of Rhizobial Infection in Lotus japonicus. Plant Physiol. 2014;165:747-58.

39. Schauser L, Wieloch W, Stougaard J. Evolution of NIN-Like Proteins in Arabidopsis, Rice, and Lotus japonicus. J Mol Evol. 2005;60:229-37.

40. Ponting CP, Ito T, Moscat J, Diaz-Meco MT, Inagaki F, Sumimoto H. OPR, PC and AID: all in the PB1 family. Trends Biochem Sci. 2002;27:10.

41. Roux B, Rodde N, Jardinaud M-F, Timmers T, Sauviac L, Cottret L, et al. An integrated analysis of plant and bacterial gene expression in symbiotic root nodules using laser-capture microdissection coupled to RNA sequencing. Plant J. 2014;77:817-37.

42. Lullien V, Barker DG, de Lajudie P, Huquet T. Plant gene expression in effective and ineffective root nodules of alfalfa (Medicago sativa). Plant Mol Biol. 1987:9:469-78.

43. Veereshlingam H, Haynes JG, Sherrier DJ, Penmetsa RV, Cook DR, Dickstein R. nip, a symbiotic Medicago truncatula mutant that forms root nodules with aberrant infection threads and plant defense-like response. Plant Physiol. 2004;136:3692-702.

44. Dellaporta SL, Wood J, Hicks JB. A plant DNA minipreparation: Version II. Plant Mol Biol Rep. 1983;1:19-21.

45. Liu Y-G, Mitsukawa N, Oosumi T, Whittier RF. Efficient isolation and mapping of Arabidopsis thaliana T-DNA insert junctions by thermal asymmetric interlaced PCR. Plant J. 1995:8:457-63.

46. Liu Y-G, Chen Y, Zhang Q. Amplification of genomic sequences flanking T-DNA insertions by thermal asymmetric interlaced polymerase chain reaction. Methods Mol Biol. 2005;286:341-8.

47. Zerbino DR, Birney E. Velvet: Algorithms for de novo short read assembly using de Bruijn graphs. Genome Res. 2008;18:821-9.

\section{Submit your next manuscript to BioMed Central and we will help you at every step:}

- We accept pre-submission inquiries

- Our selector tool helps you to find the most relevant journal

- We provide round the clock customer support

- Convenient online submission

- Thorough peer review

- Inclusion in PubMed and all major indexing services

- Maximum visibility for your research

Submit your manuscript at www.biomedcentral.com/submit
Biomed Central 